\title{
TerraSAR-X Calibration - First Results
}

\author{
Marco Schwerdt, Benjamin Bräutigam, Markus Bachmann, Björn Döring \\ Microwave and Radar Institute of the German Aerospace Center (DLR), \\ P.O.-Box 1116, D-82230 Weßling, Germany \\ Email: Marco.Schwerdt@dlr.de
}

\begin{abstract}
As TerraSAR-X, due for launch in 2007, will be an operational scientific mission with commercial potential, product quality is of crucial importance. The success or failure of the mission essentially depends on the calibration of the TerraSAR$X$ system ensuring the product quality and the correct in-orbit operation of the entire SAR system. The paper describes the inorbit calibration procedure for TerraSAR-X and dedicated activities performed during the commissioning phase. First results could not be described because TerraSAR-X was not launched up to the time of uploading the full paper.
\end{abstract}

\section{INTRODUCTION}

The essential task of calibrating TerraSAR-X is to estimate and correct systematic error contributions throughout the complete SAR system and to tie-down image information (magnitude and phase) to reference units in geophysical terms. The quality of this calibration process depends on the inherent stability of the radar system and the capability to determine and monitor the radiometric and geometric characteristics [1].

TerraSAR-X is designed to cover a wide range of swath positions and to operate in different operation modes [2] by switching the instrument over a multitude of different antenna beams (more than 12,000). Due to this high degree of flexibility of TerraSAR-X (StripMap, Spotlight, ScanSAR, right/left looking, etc.) and a tight performance with an absolute radiometric accuracy better than $1 \mathrm{~dB}$ it becomes clear, that a conventional calibration approach is not feasible, e.g. the real measurement of all antenna beams in all operation modes, as performed for the ASAR instrument of ENVISAT across the rainforest [3]. Hence, a new, more efficient, and affordable calibration concept has been developed [4], [5]. The key element of this calibration concept is a novel antenna model approach [6].

Furthermore, to account for the restricted time of calibration campaigns performed during the five months commissioning phase $(\mathrm{CP})$ of TerraSAR-X, the number of passes and places of test sites is optimized versus cost and time effort by calibrating several beams and polarization modes with the same test site.

The paper describes the in-orbit calibration procedure and the different activities performed during the CP of TerraSAR$X$. First results could not be described by the paper because TerraSAR-X was not launched up to the time of uploading the full paper.

\section{SCOPE}

Applying the novel antenna model approach the objective of in-orbit calibration can be sub-divided into four major tasks performed during commissioning of TerraSAR-X:
- Antenna Model Verification, to ensure the provision of the antenna patterns of all operation modes and the gain offset between adjoining beams.

- Geometric Calibration, to assign the SAR data to the geographic location on earth surface.

- Relative Radiometric Calibration, for the radiometric correction of SAR data within an illuminated scene.

- Absolute Radiometric Calibration, by measuring the SAR system against standard targets with well known geophysical characteristics.

\section{STRATEGY}

In addition to the complexity of TerraSAR-X and the corresponding challenge described before, the main goal after launch of the satellite is to provide calibrated and verified SAR data products as soon as possible. Thus, a strategy for an efficient but robust calibration approach has been developed. The successive baseline calibration processes are:

1) Geometric Calibration,

2) Antenna Pointing Determination,

3) Antenna Model Verification,

4) Relative Radiometric Calibration,

5) Absolute Radiometric Calibration.

Furthermore, by applying the PN-Gating Method [7], [8] the actual antenna settings will be derived in orbit. In the following the process steps of the in-orbit calibration are described in detail.

\section{A. Geometric Calibration}

The purpose of the geometric calibration is the geometric assignment of the SAR system to the earth surface. Two effects can influence the correct localization of the product:

- systematic azimuth shifts and

- the internal electronic delay of the instrument.

For both effects, corner reflectors are deployed as they have no additional electronic delay and consequently no additional source of error occurs.

\section{B. Antenna Pointing}

An important task is the determination of beam pointing errors coming from mechanical and electrical antenna misspointing as well as attitude control offsets. These errors are measured in elevation and in azimuth using an appropriate 
antenna pattern over rain forest and by ground receivers. But also a Doppler analysis is performed to evaluate a squint in flight direction.

\section{Antenna Model Verification}

The characterisation of the antenna is based on a precise antenna model, which was developed before launch considering different input parameters like:

- the geometry of the antenna,

- the excitation coefficients of all $384 \mathrm{~T} / \mathrm{R}$ modules,

- a drift and failure matrix and

- embedded radiation patterns measured on ground from single sub-arrays of the antenna.

This antenna model was validated against near field patterns precisely measured on-ground before launch. The strategy was based on the comparison between simulations and measurements. For this purpose, first of all the antenna patterns of sub-arrays were measured whereby the antenna model was loaded with these so called embedded radiation patterns. Then, one level upon, groups of these sub-arrays were measured and compared with that derived by the model. In case of TerraSAR-X the model was validated on panel level with 32 sub-arrays and on leaf level with 4 panels (= 128 subarrays). One example of the validation on-ground performed by Astrium is shown in Fig. 1. The blue curve is the measured

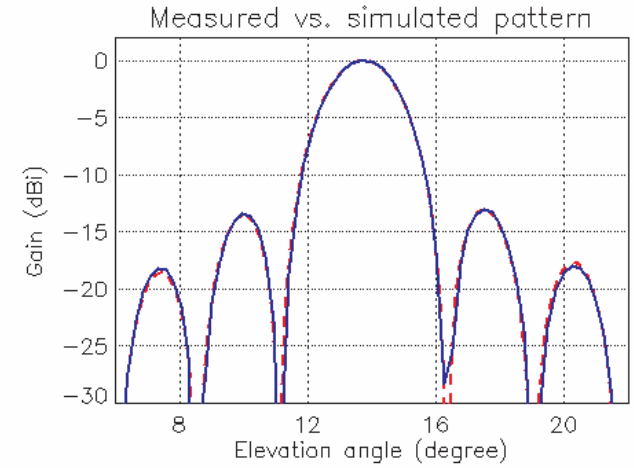

A)

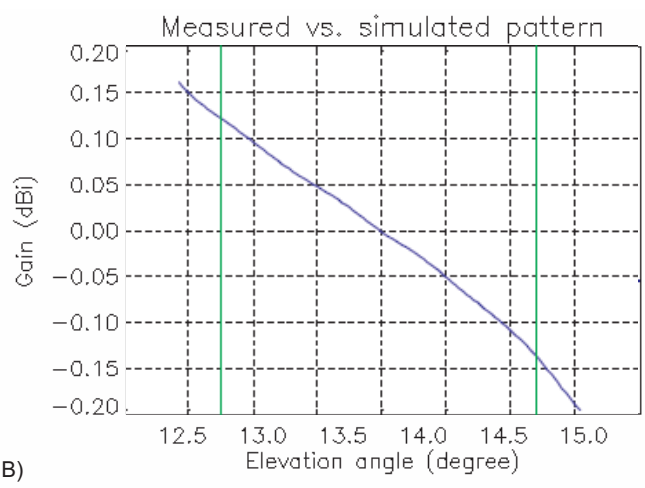

Fig. 1. A) comparison between an exemplary beam measured on ground (blue/solied) and derived by the antenna model (red/dotted), B) difference within the main beam between model and measurement.

elevation pattern of one panel and the red curve of that derived by the model. The deviation of the shape within the main lobe is less than $0.15 \mathrm{~dB}$. The results of the validation of the antenna model are described in [6].

The in-orbit verification will be performed by three selected beams with low, medium and high incidence angle. For this, the elevation patterns are measured over rain forest and compared to pre-calculated patterns. Furthermore, the transmitted pulses are recorded by deployed ground receivers during an over-flight. Thus, the transmit pattern of the TerraSAR-X antenna can be really measured in-flight verifying the oneway azimuth patterns. One example of in-flight measured antenna patterns by ground receivers is shown in Fig. 2. This measurement was performed for the ASAR instrument of ENVISAT in ScanSAR operation. The reduced amplitudes indicate the switching of the instrument from beam to beam and the envelopes represent the one-way azimuth pattern of the corresponding beam.

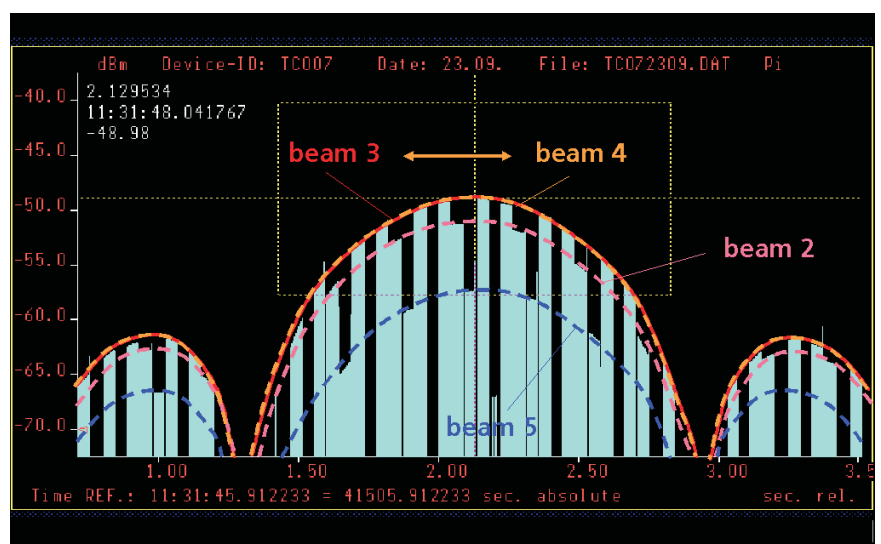

Fig. 2. One-way (transmit) azimuth antenna patterns of the ASAR/ENVISAT instrument in ScanSAR operation measured by a ground receiver

The antenna model provides a software tool that accurately determines all beam patterns based on detailed characterization of the radar antenna and knowledge of the antenna control parameters. An important input to the model are the actual beam coefficients of the active phased array antenna which can be characterized in-orbit using the novel PN-Gating method [7].

Beyond these capabilities the antenna module also features a tool for generating optimized beam coefficients under given constraints [9]. The major advantage of this beam optimization is the dynamic re-calibration in the event of instrument degradations like drifting and/or failed T/R modules. Thus, the performance of TerraSAR-X can be ensured during the lifetime of the instrument.

\section{Relative and Absolute Radiometric Calibration}

After the in-orbit verification of the antenna model thousands of beam patterns required for the relative radiometric correction during SAR data processing will be derived by the antenna model for all operation modes and all incidence angles.

In order to reduce calibration effort further on, the absolute radiometric calibration is likewise based on the antenna model, 
i.e. the real measurements over point targets with known radar cross section are performed on a reduced set of beams. The absolute calibration factors of all other beams and modes are derived via the antenna model by applying the beam to beam gain offset.

Hence, by applying the antenna model, the effort for both the relative and the absolute radiometric calibration can be significantly reduced ensuring a short duration of the $\mathrm{CP}$ and thus an early product release.

\section{Calibration Campaigns}

To ensure a successful execution of the different calibration procedures described above, dedicated calibration campaigns must be performed. This so called external calibration [10] is based on SAR data acquisition over test sites with well-known calibration targets. Basically, these sites can be homogenous areas like the Amazonian rain forest or sites with deployed point targets, like corner reflectors, active transponders or ground receivers. The most important driver to plan and coordinate these activities is the coverage of TerraSAR-X, because the coverage defines the number of feasible measurements and drives consequently the schedule.

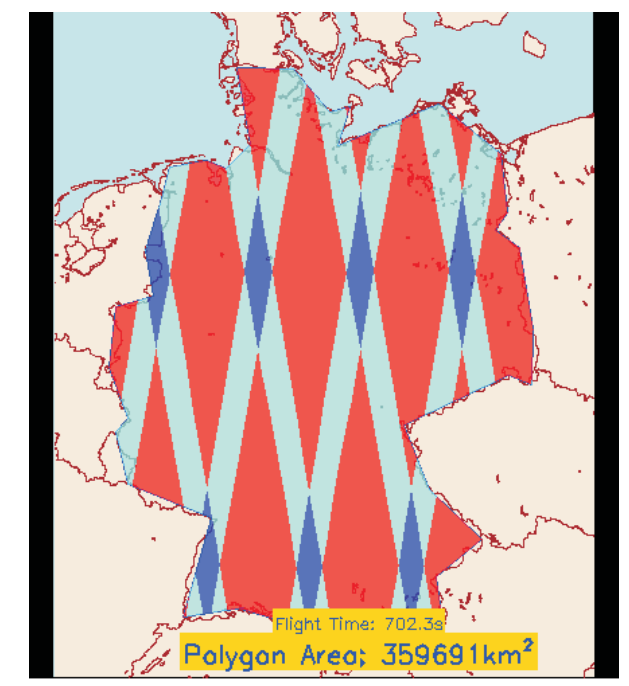

Fig. 3. Coverage of TerraSAR-X StripMap beam 11 within a complete repeat cycle of 11 days, red areas: no coverage, light blue areas: covered once and dark blue area: covered twice.

\section{A. Coverage of TerraSAR-X}

The coverage on earth surface needs to be evaluated with respect to the required number of point target and rain forest measurements. As a single beam does not cover the complete globe, the selection of test sites is constrained to the availability of respective beams on ground. Fig. 3 shows the coverage of the full performance beam 11 over Germany in right looking mode for the repeat cycle of TerraSAR-X with 11 days. In order to obtain as many overflights as possible across deployed calibration targets, special test site configurations have been established in the cross over points of ascending and descending swathes.

\section{B. Test Site Configuration}

Driven by the radiometric accuracy budget within a StripMap swath of $30 \mathrm{~km}$ range two targets are deployed in near range, at swath centre and in far range, respectively, see Fig 4 . The targets must be sufficiently separated to avoid ambiguities in the image. Due to a slight displacement, most of the positions (red crosses) can be applied twice for descending and ascending orbit. Thus the number of required target positions within a test site could be reduced extremely.

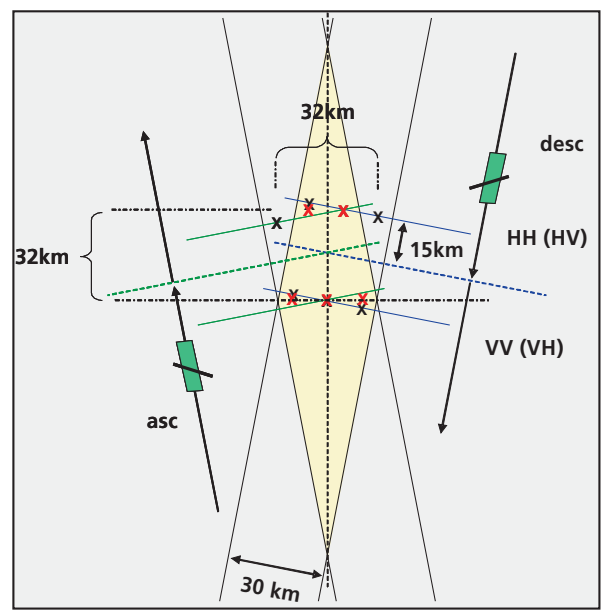

Fig. 4. Test site configuration for TerraSAR-X. The test site consists of 7 target positions and enclose an area of $32 \mathrm{~km} \times 32 \mathrm{~km}$.

Furthermore, in order to save passes for the antenna model verification by in-flight measured antenna patterns with ground receivers, two different polarizations are measured per pass by switching the instrument between the deployed ground receivers as indicated in Fig. 4 by the blue lines.

\section{DLR Calibration Field}

To achieve a high number of passes during a restricted time of commissioning TerraSAR-X a calibration field has established enclosing an area of $120 \mathrm{~km}$ x $40 \mathrm{~km}$ and consisting of 30 target positions, see Fig 5. By this calibration field several crossing over areas of the same and of different beams are covered and considering the test site configuration described above a re-use of most of the target positions for several passes (e.g. ascending and descending) is possible.

Mainly for logistic reasons this calibration field was selected in South Germany which can be operated and maintained from Oberpfaffenhofen, the location of the Microwave and Radar Institute, see Fig 5. The existing infrastructure of the institute is well prepared for executing calibration campaigns over large test sites. It should be mentioned that additional test sites are deployed in North Germany, Barcelona and Switzerland.

\section{DLR Calibration Facility}

The realization of the TerraSAR-X calibration concept and the implementation of the procedures described above requires a calibration facility that is well-equipped with ground calibration hardware as well as software tools for evaluating the 


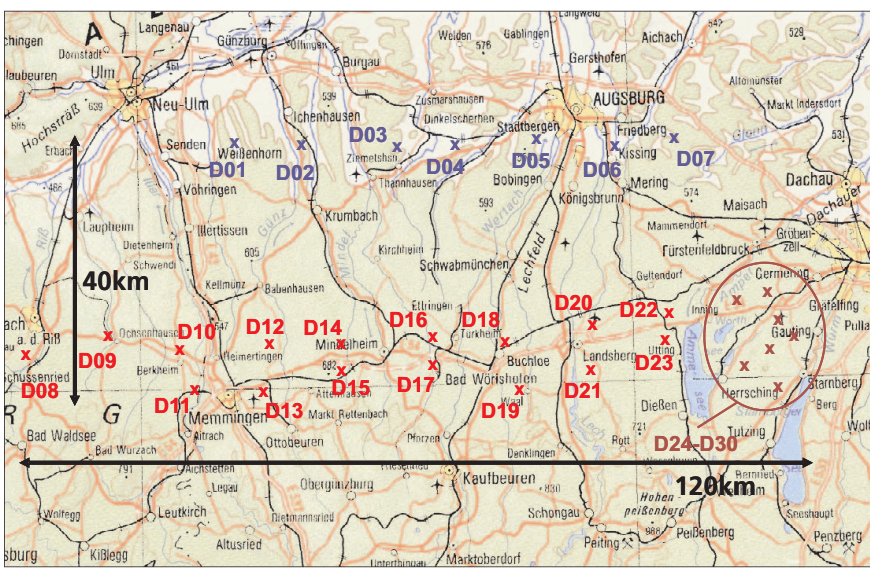

Fig. 5. DLR's Calibration Field in South Germany consisting of 30 target positions and enclosing an area of $120 \mathrm{~km} \times 40 \mathrm{~km}$.

measurements. For this purpose the Microwave and Radar Institute has developed and established the following reliable and accurate ground equipment:

- accurate ground targets like corner reflectors and transponders precisely surveyed for geometric and radiometric calibration [11],

- ground receivers measuring the one-way azimuth pattern of the SAR antenna during an overflight [11],

- different analysis and evaluation tools, like that of

- the mentioned antenna model providing an estimation of the actual antenna pattern,

- CALIX, a software tool for point target analysis, featuring measurements of impulse response function parameters and the absolute calibration factor. Furthermore a geometric analysis for accurately surveyed targets can be performed yielding the internal delay of the instrument and the datation accuracy. Figure 6 shows a screen-shot of this tool.

\section{CONCLUSION}

In preparation of the TerraSAR-X satellite launch an efficient but accurate strategy has been established to calibrate the whole TerraSAR-X system in a restricted time for performing different calibration procedures.

By applying the antenna model as key element of calibrating TerraSAR-X, most of the antenna characterization is shifted from the commissioning phase to pre-launch activities. Consequently, after launch only a few selected beams have to be really measured in-flight, ensuring a short duration of the commissioning phase for early product release, i.e. for operational readiness as early as possible.

Furthermore, a multitude of powerful software tools has been developed within on-ground tests to support the evaluation of calibration measurements. The evaluation of data acquired during the calibration campaigns yields different calibration parameters like the antenna pattern or the absolute calibration factor. Therefore, suitable test site configurations have been established, supported by precise and sophisticated ground equipment from the DLR calibration facility.

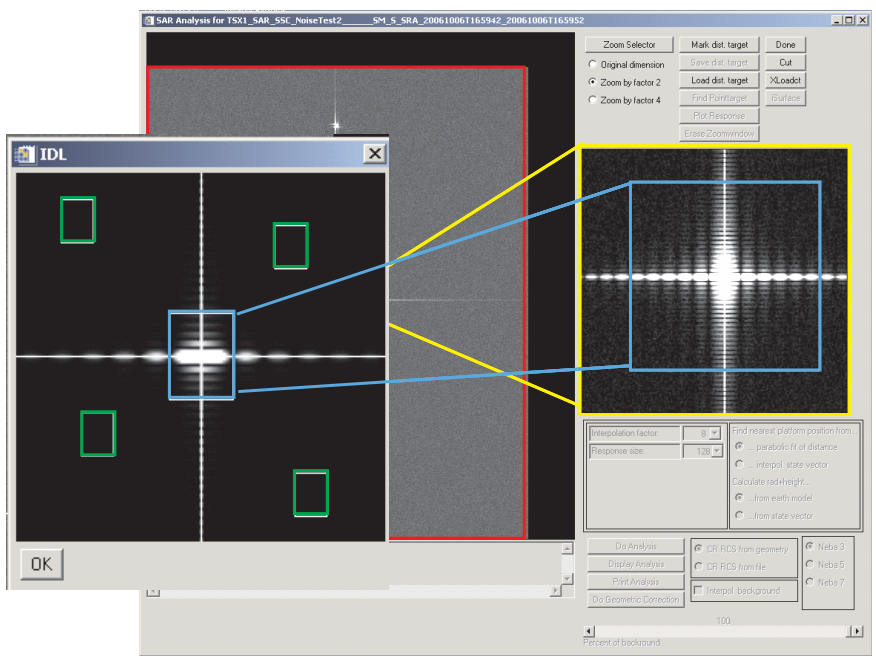

Fig. 6. Screen-shot of CALIX, a S/W tool for point target and geometric analysis.

Keeping the cost affordable is the major challenge of calibrating future SAR sensors. e.g. like TanDEM-X [12], and the described calibration strategy using innovative methods like the applied antenna model approach and the PN-Gating method make an important contribution to achieving this aim.

\section{REFERENCES}

[1] M. Schwerdt and D. Hounam, "Quality Control and Calibration of Future SAR Systems," in ODAS 2001, 3rd ONERA-DLR Aerospace Symposium, Paris, France, 2001, pp. S6-6.

[2] R. Werninghaus, "The TerraSAR-X Mission," in 6th European Conference on Synthetic Aperture Radar, Dresden, Germany, 2006.

[3] M. Zink and B. Rosich, "Antenna Elevation Pattern Estimation from Rain Forest Acquisitions," in ENVISAT/ASAR Calibration Review (ECR) of ESTEC, European Space Agency (ESA), Noordwijk, Netherland, 2002.

[4] M. Schwerdt, D. Hounam, J. L. Alvarez-Pérez, and T. Molkenthin, "The Calibration Concept of TerraSAR-X, a Multiple Mode High Resolution SAR," Canadian Journal of Remote Sensing, Vol. 31, No.1, Feb 2005.

[5] M. Schwerdt, D. Hounam, B. Bräutigam, and J. L. Alvarez-Pérez, "TerraSAR-X: Calibration Concept of a Multiple Mode High Resolution SAR," in 25th International Geoscience And Remote Sensing Symposium, Seoul, Korea, 2005.

[6] M. Bachmann, M. Schwerdt, B. Bräutigam, B. Grafmüller, A. Herschlein, and J. L. Alvarez-Pérez, "The TerraSAR-X Antenna Model Approach," in 2nd International ITG Conference on Antennas, Munich, Germany, 2007.

[7] D. Hounam, M. Schwerdt, and M. Zink, "Active Antenna Module Characterisation by Pseudo-Noise Gating," in 25th ESA Antenna Workshop on Satellite Antenna Technology, Noordwijk, Netherlands, 2002.

[8] B. Bräutigam, M. Schwerdt, M. Bachmann, and M. Stangl, "Individual T/R Module Characterisation of the TerraSAR-X Active Phased Array Antenna by Calibration Pulse Sequences with Orthogonal Codes," in 26th International Geoscience And Remote Sensing Symposium, Barcelona, Spain, 2007

[9] J. L. Alvarez-Pérez, M. Schwerdt, D. Hounam, R. Torres, C. Buck, and M. Zink, "Antenna Pattern Optimization for the ENVISAT ASAR Antenna with Failed Elements," in 25th ESA Antenna Workshop on Satellite Antenna Technology, Noordwijk, Netherlands, 2002.

[10] B. Bräutigam, M. Schwerdt, and M. Bachmann, "The External Calibration of TerraSAR-X, a Multiple Mode SAR-System," in 6th European Conference on Synthetic Aperture Radar, Dresden, Germany, 2006.

[11] B. Döring and M. Schwerdt, "TerraSAR-X Calibration Ground Equipment," in 4th European Radar Conference, Munich, Germany, 2007.

[12] J. Jueso Gonzalez, M. Bachmann, H. Fiedler, S. Huber, G. Krieger, and M. Zink, "DEM Calibration Concept of TanDEM-X," in 26th International Geoscience And Remote Sensing Symposium, Barcelona, Spain, 2007. 